\title{
Dual-pulse laser-induced spark ignition and flame propagation of a methane diffusion jet flame
}

Lydia Wermer ${ }^{\mathrm{a}, \mathrm{b}}$, James Hansson ${ }^{\mathrm{b}, \mathrm{c}}$, Seong-kyun $\operatorname{Im}^{\mathrm{a}, \mathrm{b}, *}$

${ }^{a}$ Department of Aerospace and Mechanical Engineering, University of Notre Dame, Notre Dame, IN, USA

${ }^{\mathrm{b}}$ Department of Mechanical Engineering, Worcester Polytechnic Institute, Worcester, MA, USA

${ }^{\mathrm{c}}$ Alden Research Laboratory, Inc., Holden, MA, USA

* Corresponding author. Current Address: Department of Aerospace and Mechanical Engineering, University of Notre Dame, Hessert Laboratory, Notre Dame, IN 46556, USA. Email address:

sim@nd.edu

Colloquium 14, NOVEL COMBUSTION CONCEPTS, TECHNOLOGIES AND SYSTEMS including mini- and microcombustors, catalytic combustion, mild combustion, plasma-aided combustion, hydrothermal reaction, and other novel combustion processes.

Total length of paper (Method 1):

- $\quad$ Main text: 3508

- References: 350

- Figure 1: 320

- Figure 2: 155

- Figure 3: 480

- Figure 4: 112

- Figure 5: 476

- Figure 6: 532

- Total: 5933 


\begin{abstract}
An experimental investigation was performed to study the ignition and flame propagation behaviors of a methane diffusion jet flame $(\operatorname{Re}=5500)$ when dual pulse laser-induced spark discharges were introduced in a mixing layer. Time intervals $(d t)$ of $50 \mathrm{~ns}, 100 \mu \mathrm{s}$, and $600 \mu \mathrm{s}$ between two laser pulses were tested, and the results were compared to a single pulse discharge case with the same total laser energy $(60 \mathrm{~mJ})$. The laser-induced breakdown was introduced to the mixing layer at 9.5 jet diameters downstream from the jet exit. The chemical delay time scale under the flow condition was approximately $350 \mu \mathrm{s}\left(t_{c d}\right)$. The disturbances in density fields generated by laser-induced breakdowns and chemical reactions were visualized using a high-speed schlieren imaging technique. The visualization at stationary air showed that interactions between two laser-induced breakdowns increased the surface area of hot plumes, but the effects of the interactions diminished when the breakdowns were introduced in a non-reacting air jet. The effects of the flow on the hot plumes prevailed the interaction effects between two breakdowns in non-reacting air jet condition. However, when the dual pulse laserinduced spark discharges $\left(d t=600 \mu \mathrm{s}, d t / t_{c d}=1.71\right)$ were generated in a mixing layer of a methane jet, a rapid propagation of the flame was observed since the second breakdown enlarged the ignition kernel surfaces generated by the first breakdown. The results showed that using the dual pulse with intervals shorter than the electron lifetime scale (under $200 \mathrm{ns)}$ or longer than the chemical delay time scale could be beneficial in enhancing ignition and flame propagation processes due to the increased energy deposition or hot surface area, respectively.
\end{abstract}

Keywords: Laser-induced breakdown; Ignition; Diffusion flame; Schlieren imaging 


\section{Introduction}

Laser-induced spark (LIS) ignition has many potential benefits such as low heat loss and combustion control over the timing and the location of ignition [1-5]. The LIS is driven by the multi-photon ionization of gas molecules. Then, electrons from the initial ionization collide with other molecules, resulting in electron avalanches and gas breakdowns [4-6]. Consequently, the LIS produces highly reactive intermediate species at high temperature and pressure conditions. The LIS initiates the ignition kernels that undergo a transition to flame propagation eventually $[7,8]$. Unlike conventional electrode spark discharges, LIS plasma discharges can be formed by focusing the laser beam on any location within a combustor. Therefore, the heat loss due to electrodes can be reduced significantly $[9,10]$.

Although the LIS ignition has potential advantages in combustion control, there are a few issues hindering the use of the laser spark ignition in practices. One of the major issues is the energy loss through a strong blast wave induced by the rapid laser energy deposition. A significant amount of energy absorbed by the plasma discharge $(50-70 \%)$ is consumed by the blast wave propagation during the early stage of the breakdown (order of microsecond), and the energy carried over by the blast wave cannot be used for the ignition occurring between hundreds of microseconds and a few milliseconds after the breakdown $[11,12]$. Therefore, it is critical to minimize the loss through the blast wave, leading to more efficient ignition processes.

In the current study, we investigated the use of dual-pulse laser-induced spark (DPLIS) on the ignition of methane diffusion jet flame, which is a potential way to recover the energy of the blast wave. The DPLIS has usually been used to enhance the plasma emission intensity in laser-induced breakdown spectroscopy (LIBS) $[13,14]$. The first laser pulse is focused on the target gas medium, generating a breakdown. Once the electron number density reaches a certain level, the plasma discharge becomes optically opaque. Then, the plasma discharge absorbs the photon energy from the second laser pulse. Since the plasma discharge is optically opaque, the absorption rate increases through the inverse 
Bremsstrahlung absorption [15-17]. As a result, DPLIS generates higher temperature and larger spark discharge than a single laser if the time interval between two pulses is set adequately (under a couple of hundred nanoseconds). Therefore, the energy loss through the blast wave can be reduced when DPLIS is used by comparing with single laser pulse with the same total incident energy. However, when the electron number density of the plasma discharge induced by the first laser pulse decays and the density of the medium decreases due to the thermal expansion, the medium does not absorb the photon energy of the second laser pulse. Consequently, there occurs a so-called "transparent interval" between a couple of hundred nanoseconds and ten microseconds [15]. After the plasma discharge of the first laser pulse cools down, the second laser pulse begins to generate a breakdown again (after ten microseconds). At this time intervals, the blast wave induced by the second breakdown strongly interacts with the expanding hot plume generated by the first breakdown, increasing the surface area of expanding hot plumes [17]. The increased surface area of the hot plumes can be beneficial to enhance the ignition and flame propagation processes. The interactions between two successive laser pulses at these time intervals are the particular interests of the current study. There have been few studies using two laser pulses for flame ignition. However, the studies focused primarily on pre-heating or -ionizing from the first laser pulse without breakdowns [12,18]. The present study demonstrates a new approach, the use of DPLIS with time intervals under a hundred nanosecond or over a hundred microsecond on flame ignition and propagation to reduce or recover the energy consumed by the blast waves.

\section{Experimental Setup}

The experimental setup consisted of a diffusion burner with a co-flow configuration, an LIS generation system, and a high-speed schlieren imaging system, as schematically depicted in Fig. 1a. The diffusion burner had a $3 \mathrm{~mm}$ inner diameter $\left(d_{j}\right)$ fuel jet nozzle and a $250 \mathrm{~mm}$ diameter annular air co-flow. The bulk speed of the fuel jet was set to $30 \mathrm{~m} / \mathrm{s}(\operatorname{Re}=5500)$ by controlling the fuel flow rate 
using a mass flow controller (Sierra Instruments, Inc, SmartTrak 100). A fan blower delivered the coflowing air of $0.3 \mathrm{~m} / \mathrm{s}$ flow speed at the exit. Several screens and a honeycomb were inserted in the annular co-flow to ensure a uniform exit speed distribution and minimize flow fluctuations at the exit. To generate LIS discharges at desired locations, laser beams of a frequency-doubled dual cavity Nd:YAG laser (New Wave Research, Gemini 400, 532 nm maximum 200 mJ/pulse, 10 ns pulse width) were focused by using a focusing lens $(f=150 \mathrm{~mm})$. Each laser pulse had the energy of $30 \mathrm{~mJ} / \mathrm{pulse}$, and thus, the total laser energy was fixed at $60 \mathrm{~mJ}$. Intervals $(d t)$ between the two laser pulses were adjusted by using a pulse generator (BNC, Model 577-8C). When a single pulse was tested, the second laser pulse was deactivated. The energy of each laser beam was monitored and recorded by using a laser power meter (Ophir, PE50BF-DIFH-C). The laser energy at two different locations, the upstream and downstream of the LIS, was measured to estimate the energy deposition from the laser beams to LIS discharges. The energy over 200 pulses was averaged and the nominal fluctuation of the laser energy was about $7 \%$. The disturbed density fields by the LIS, fuel jet, and flame were visualized by a conventional dual-field lens high-speed schlieren imaging system consisting of an incoherent laser light from a high-speed diode laser (Cavitar, Cavilux Smart $640 \mathrm{~nm}$ ), optical lenses, a knife-edge, and a high speed camera (Photron, Fastcam APX Ultima) equipped with a bandpass filter $(640 \mathrm{~nm} \pm 12 \mathrm{~nm}$

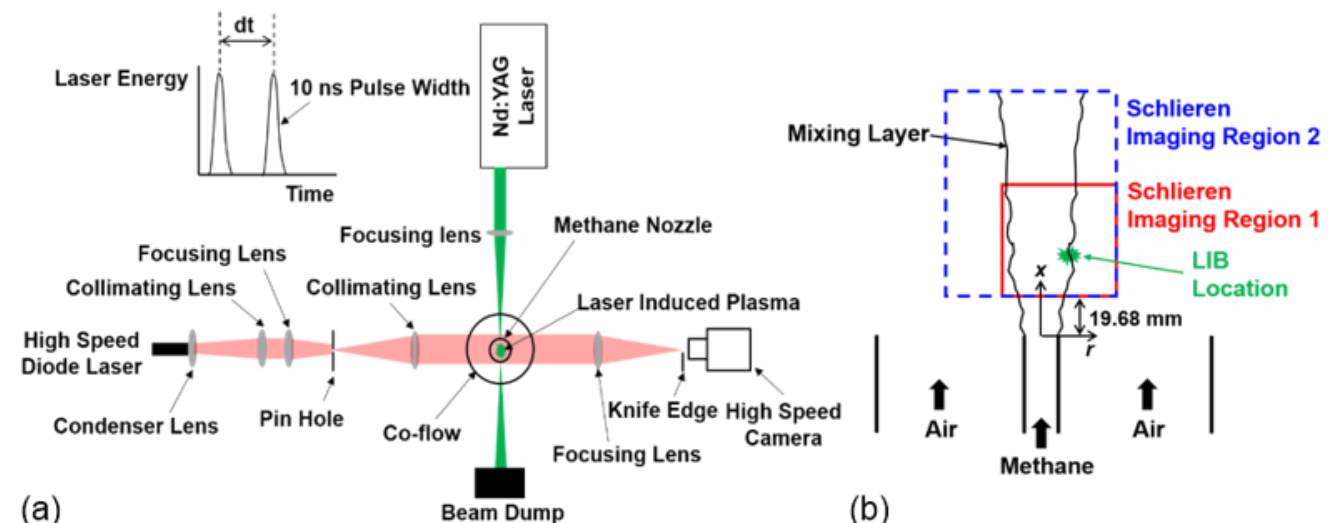

Figure 1. Schematics of (a) overall experimental setup and (b) schlieren imaging regions and spark discharge location, $r$ : radial direction from the jet center and $x$ : axial direction from the jet exit 
FWHM). The diode laser light was first focused onto a 1-mm-diameter pinhole using a lens system to create a point light source. This light was then collimated into a 75-mm-diameter circular field of view using a schlieren field lens $(f=500 \mathrm{~mm})$, while its path in the schlieren imaging system was aligned perpendicularly to the Nd:YAG laser beam path. After passing through the region of interest, the light was focused onto a knife edge using a focusing lens $(f=500 \mathrm{~mm})$, and the final image was recorded using the high-speed camera equipped with an imaging lens (Nikon, Mico-Nikkor, $f=50 \mathrm{~mm}$ ). A frame rate of $10 \mathrm{kHz}$ was used to resolve the time evolution of the LIS, ignition, and flame propagation. The imaging system was enabled to freeze the flow features for capturing sharp images by using the $10 \mathrm{~ns}$ pulse width of the diode laser. The LIS generation and imaging systems were synchronized by using the pulse generator (BNC, Model 577-8C).

The LIS discharge location and the schlieren visualization regions are schematically shown in Fig. 1b. The LIS was introduced at $28.5 \mathrm{~mm}$ downstream of the jet exit $(x / d j=9.5, x$ : axial coordinate $)$ and $3.7 \mathrm{~mm}$ from the center axis of the fuel nozzle in radial direction $(r / r j=2.47, r$ : radial coordinate, $r j$ : radius of the jet) where was an ignitable mixing layer. The chemical delay time scale $\left(t_{c d}=350 \mu \mathrm{s}\right)$ was determined by examining schlieren images. The first schlieren region was used to visualize the evolution of the LIS discharges ( $1 \mu$ s to $1 \mathrm{~ms}$ after the first laser pulse) and the early stage of flame ignition (up to $2 \mathrm{~ms}$ after the first laser pulse). The size of the visualization window was $22.58 \mathrm{~mm} \times$ $22.58 \mathrm{~mm}$. The second schlieren region visualized the propagation of the flame from $6 \mathrm{~ms}$ to $60 \mathrm{~ms}$ after the first laser pulse. The size of the visualization region was $31.88 \mathrm{~mm} \times 46.84 \mathrm{~mm}$. Both of the regions were visualized at $19.68 \mathrm{~mm}$ downstream from the fuel nozzle exit $(x / d j=6.56)$, and the spatial resolution was $158.8 \mu \mathrm{m} /$ pixel.

\section{Results and Discussion}

\subsection{Laser energy measurements}


The energy absorption of the second laser pulse at both air and methane jets with a range of pulse intervals $(d t)$ is shown in Fig. 2. The second laser pulse absorption increased beyond that of single laser pulse absorption rate $(60 \%)$ when the pulse intervals less than 200 ns were used. The LIS was optically opaque due to the high electron number density. As a result, the absorption rate increased through inverse Bremsstrahlung absorption. However, the absorption of the second laser pulse decreased rapidly when the electron density decayed approximately $300 \mathrm{~ns}$ after the first laser pulse. The absorption of the second laser pulse remained low $(<20 \%)$ until the pulse interval became $10 \mu$ s. Then, the absorption of the second laser pulse recovered to the single laser pulse absorption. Both of the air and methane jet cases exhibited very similar trends, but the methane jet case had a lower absorption than the air jet case at the intervals between $350 \mu$ s and $800 \mu \mathrm{s}\left(d t / t_{c d}=1\right.$ and 2.29). The lower absorption was caused by the chemical reaction that decreased the gas density at the LIS location. Then again, the absorption of the second laser pulse recovered to the single laser pulse absorption rate when the mixture at the LIS location was refreshed $\left(d t / t_{c d}>2\right)$. Based on the absorption energy measurement results, three intervals were chosen to investigate the effects of the intervals between the two laser pulses on ignition and the flame propagation of the methane turbulent diffusion flame. The first case was $d t=50 \mathrm{~ns}$ that had a higher absorption rate of the second laser pulse than the single laser pulse. The second case was $d t=$

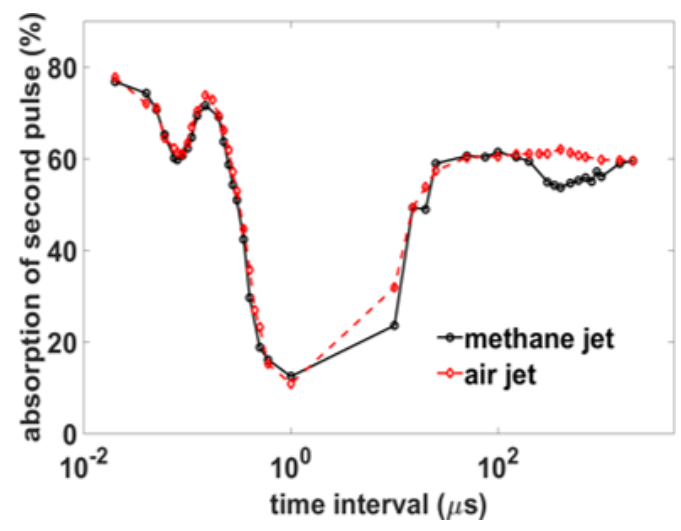

Figure 2. Spark energy absorption of second laser pulse in air (red dashed line) and methane (black solid line) flows 
$100 \mu \mathrm{s}\left(d t / t_{c d}=0.29\right)$ where the absorption recovered from the transparent condition before ignition timing. The last case was $d t=600 \mu \mathrm{s}\left(d t / t_{c d}=1.71\right)$ in which the ignition was in process. The three different cases were compared to the single laser pulse case with the same total laser energy $(60 \mathrm{~mJ})$.

\subsection{LIS at stationary and flowing air}

The flow and density fields generated by the DPLIS were visualized in non-reacting stationary and flowing conditions to investigate general flow features without heat release. Figure 3 shows the timesequential schlieren images of the single pulse and the dual pulse of three different time intervals $(d t=$ $50 \mathrm{~ns}, 100 \mu \mathrm{s}$, and $600 \mu \mathrm{s}$ ). For imaging, the camera frame rate of $50 \mathrm{kHz}$ was used, and the width of each image was $10.16 \mathrm{~mm}$. The time noted at the top of each column represents the time after the first LIS.

The overall features of disturbed density fields by the LIS were very similar between the single pulse and the dual pulse of $50 \mathrm{~ns}$ interval (the first two rows of the images). Shortly after the breakdown, there was a shockwave propagating away from the discharge as seen in the $5 \mu$ s time frame. Then, the heated gas expanded the perpendicular direction (vertical) to the incident laser (horizontal). The third

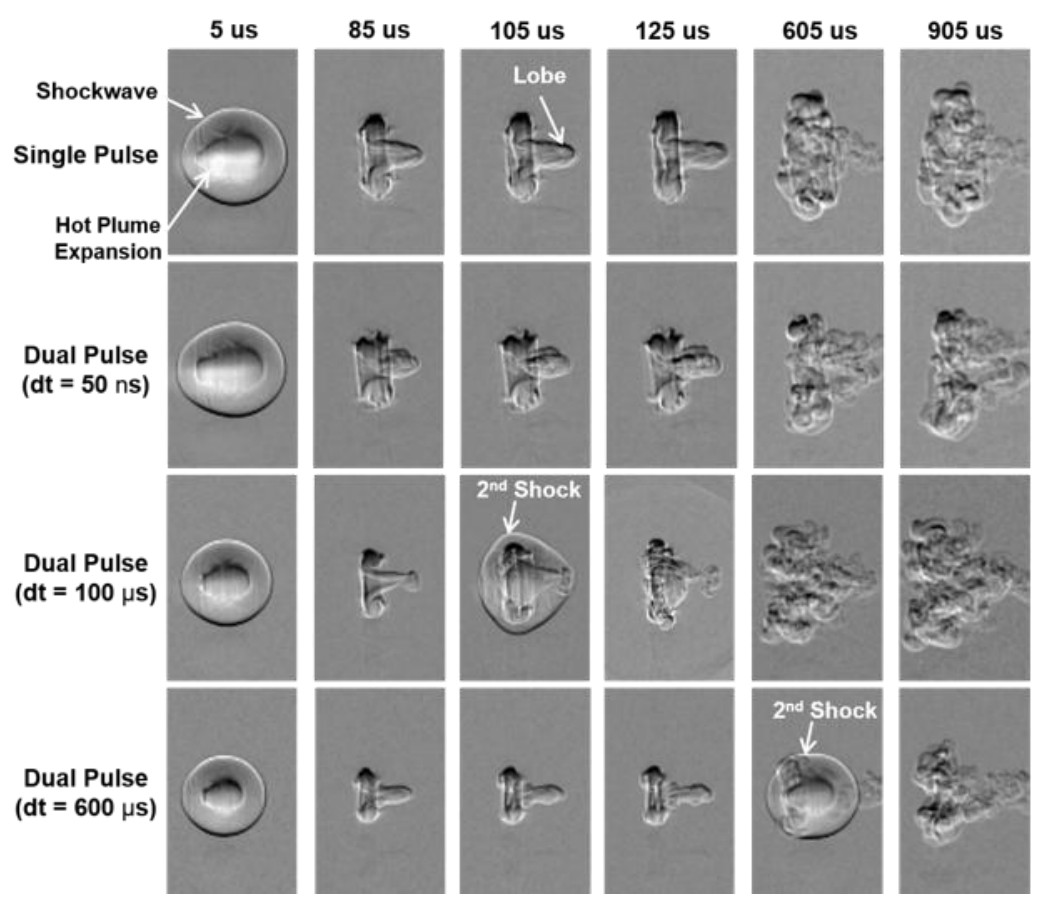

Figure 3. Time-sequential schlieren images of single pulse and dual pulse of three different time intervals $(\mathrm{dt}=50 \mathrm{~ns}, 100 \mu \mathrm{s}$, and $600 \mu \mathrm{s})$, incident laser from the right side of the images 
lobe propagation to the reverse direction of the incident laser was also observed ( $85 \mu \mathrm{s}$ to $105 \mu \mathrm{s})$. The expansion of the hot plume generated by LIS provoked the thermal instability at the propagating surfaces. Consequently, the expansion induced eddy structures as seen in $605 \mu$ s and $905 \mu$ s time frames. Since the total energy absorption in the dual pulse of $d t=50 \mathrm{~ns}$ case was higher than the single pulse case, the overall length of the hot plume induced by dual pulse LIS was also longer. Also, the DPLIS triggered the instability of the propagating surface faster than did the single pulse, leading to the larger surface area of the hot plume.

The first LIS discharge sizes of $100 \mu$ s and $600 \mu$ s dual pulse interval cases (two of the bottom rows of Fig. 3) were smaller than the first two cases (single pulse and dual pulse of $d t=50 \mathrm{~ns}$ ) because only half of the total energy was used to generate the first breakdown. However, the overall process of hot plume expansion remained self-similar until the second breakdown was induced. The hot plume expanded vertically and generated the third lobe. Then, the expanding hot plume interacted strongly with the shockwave induced by the second LIS as depicted in $105 \mu$ s time frame of the dual pulse of $\mathrm{dt}=$ $100 \mu$ s case (third row of Fig. 3). The shape of the shockwave was strongly distorted due to the disturbed density field from the first LIS. Further, the Richtmyer-Meshkov instability occurred when the shockwave generated by the second breakdown passed through the surface of the hot plume produced by the first breakdown [12]. The interaction led to creating finer eddies along with the increased overall surface area that could be beneficial for mixing and flame propagation.

The second LIS was gradually decoupled from the first LIS as the temperature gradient at the hot plume surface decreased due to the expansion. As shown in the dual pulse images of $d t=600 \mu \mathrm{s}$ case (last row of Fig. 3), the shape of the shockwave induced by the second LIS became circular (or spherical), indicating that the effects of the first hot plume on the second LIS were not significant. As a result, the disturbed density field seemed to be the superposition of the hot plumes from the two distinct pulses. 
Figure 4 represents the schlieren images of the disturbed density fields by the LIS discharges in schlieren imaging region 1 (Fig. 1b). The air jet of $30 \mathrm{~m} / \mathrm{s}$ bulk speed at the jet exit was used, and the LIS discharges were introduced at a shear layer as depicted in Fig. 1b. The images were taken after 2 ms from the first laser pulse. As shown in Fig. 4, there was a strong interaction between the air jet and the density field disturbed by the LIS discharge. As a result, the eddies induced by the LIS (see Fig. 3) cascaded into finer-scale eddies. Unlike the stationary condition (Fig. 3) and the reacting flow condition which will be presented later in the paper, the overall size of the disturbed density field and the size of the distribution of the eddies were similar between the four different conditions. It seemed that the effects of the flow prevailed the effects of pulse-to-pulse coupling of the DPLIS on the disturbed density

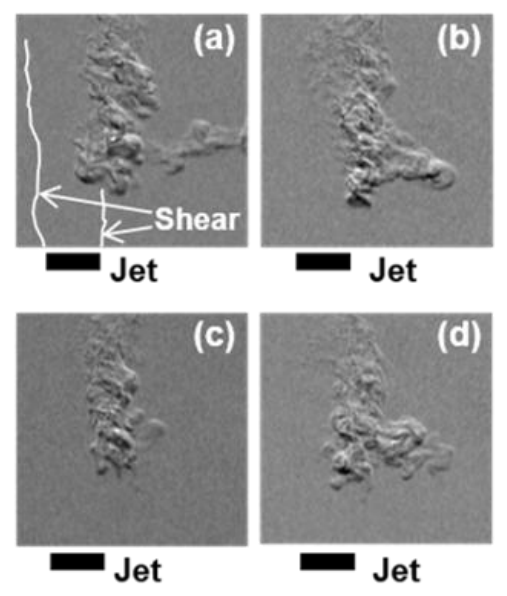

Figure 4. Schlieren images of (a) single pulse, dual pulse of (b) $50 \mathrm{~ns}$, (c) $100 \mu \mathrm{s}$, and (d) $600 \mu \mathrm{s}$ intervals after $2 \mathrm{~ms}$ from the first laser pulse, incident laser from the right side of the images field in the non-reacting jet condition.

\subsection{Ignition and flame propagation by LIS}

The flow features of flame ignition and propagation were visualized, as shown in Fig. 5. Schlieren imaging region 1 (Fig. 1b) was used to capture the early stages of ignition processes when the LIS discharges were introduced at the shear layer of a methane jet. As depicted in Fig. 5, the hot plume expansion and cascading eddy structures into finer scales were observed similar to the stationary and non-reaction flow cases. One noticeable difference in a methane jet case was the distribution of the 
eddy sizes. Since ignition occurred approximately $350 \mu$ s (Fig. 2), heat release from the chemical reactions began to influence the flow features. Due to heat release and expansion, the local Reynolds number decreased. Consequently, the finer scales of the disturbed density field disappeared. This difference was clearly seen when the images of Fig. 5 at 2 ms time frame were compared to the images of Fig. 4. The small scale eddies at the outside mixing layer were vanished due to the heat release and expansion.

The overall flow features in the dual pulse of $d t=50 \mathrm{~ns}$ and $d t=100 \mu \mathrm{s}$ interval cases were similar to those of the single pulse case. However, the affected areas in the dual pulse of $d t=50 \mathrm{~ns}$ and $d t=$ $100 \mu$ s interval cases were larger and smaller than the single pulse case, respectively. In the dual pulse of $d t=50 \mathrm{~ns}$ case, the higher energy was deposited into the LIS discharge, leading to faster ignition and flame propagation. As a result, the size of the affected area from heat release became larger comparing to the single pulse case at the same time frame. In the dual pulse of $d t=100 \mu$ s case, the energy deposited into the first LIS discharge was smaller than the single pulse case. Furthermore, the effects of

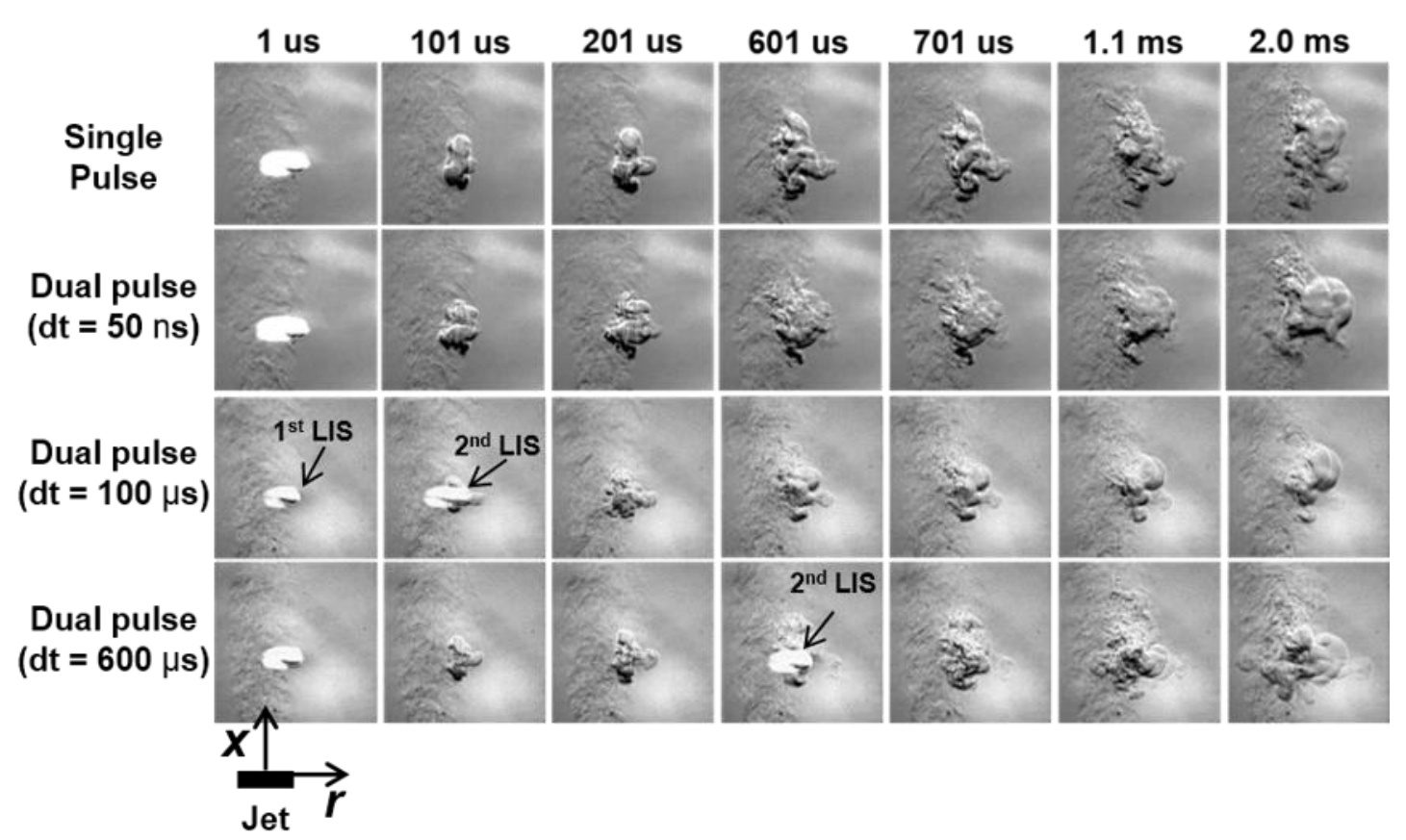

Figure 5. Schlieren images of early stages of the ignition induced by single pulse, dual pulse of $50 \mathrm{~ns}, 100 \mu \mathrm{s}$, and $600 \mu$ s intervals, incident laser from the right side of the images (Schlieren imaging region 1). Relative location of the jet exit and coordinates are noted at the bottom left image. 
the increased surface area from the second LIS discharge on ignition became minimal since the flow effect prevails. Consequently, the size of ignition kernels was smaller than other two previous cases.

The effect of the second LIS discharge on flame propagation, however, became significant when the interval between laser pulses increased to $600 \mu \mathrm{s}\left(d t / t_{c d}=1.71\right)$ as seen in Fig. 5. At $600 \mu$ s time frame, the ignition process was ongoing, and the process generated strong density gradients at the surfaces of the expanding ignition kernels. When the second LIS was introduced, the shockwave and the hot plume induced by the second LIS interacted with the expanding ignition kernel surfaces. The interactions were strong enough to propagate the ignition kernels to the other side of the jet as seen in 2 ms time frame image of the last row in Fig. 5. The propagation enlarged the area of the contact surface between mixing layer and ignition kernels.

The effect of the enlarged contact surface on flame propagation is clearly visualized in Fig. 6 that

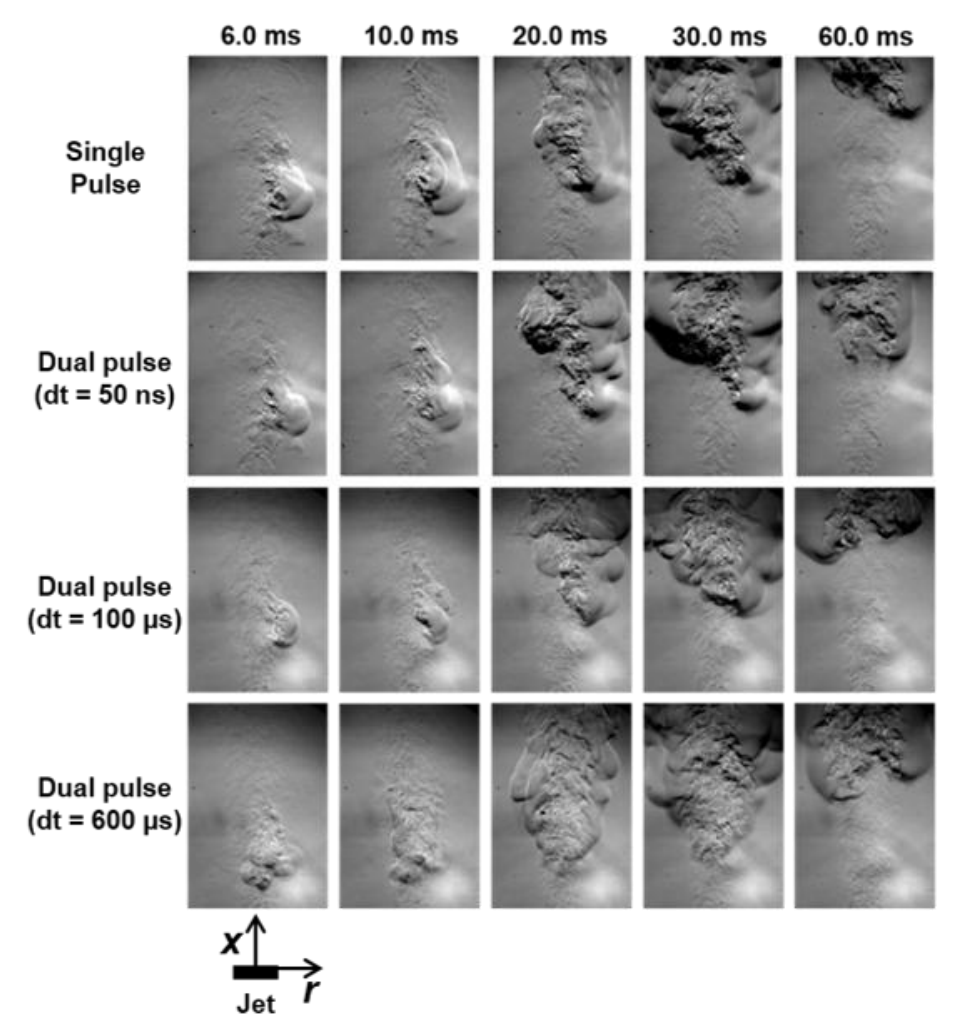

Figure 6. Schlieren images of flame propagation of single pulse, dual pulse of $50 \mathrm{~ns}, 100 \mu \mathrm{s}$, and $600 \mu \mathrm{s}$ intervals (Schlieren imaging region 2). Relative location of the jet exit and coordinates are noted at the bottom left image. 
depicts the flow features in schlieren imaging region 2 (Fig. 1b) from $6 \mathrm{~ms}$ to $60 \mathrm{~ms}$ after the first laser pulse. Since the ignition source (LIS discharge) was introduced to the one side of the mixing layer, the flame initially propagated along the side of the mixing layer. Then, the flame ignited the adjacent combustible mixture, leading to the lateral flame propagation. Due to the finite speed of the fuel jet, the lateral propagation was eventually bent to an oblique direction from the ignition source as shown in the first three rows of Fig. 6. On the other hand, the flame propagation sequence in the dual pulse of $d t=$ $600 \mu$ s case exhibited different behaviors than in the other three cases. Due to the laterally enlarged ignition kernels depicted in Fig. 5, a simultaneous ignition of the diffusion flame across the jet was observed as seen in the last row of Fig. 6, leading to the flat flame base. Therefore, the flame base location of $d t=600 \mu$ s case was relatively lower than the other three cases at the same time frame.

\section{Conclusion}

The ignition and flame propagation of a methane diffusion jet flame induced by DPLIS discharges were investigated at a co-flow configuration using a high-speed schlieren imaging system. The bulk jet speed of $30 \mathrm{~m} / \mathrm{s}(\operatorname{Re}=5500)$ was studied at $0.3 \mathrm{~m} / \mathrm{s}$ co-flow speed. Based on the results of the laser energy measurement and schlieren visualization at a stationary air, three different interval regimes between two laser pulses that were potentially beneficial for ignition and flame propagation were selected in the current study. The first time interval was $50 \mathrm{~ns}$; it increased the energy deposition to the LIS discharge and provoked the instability of propagating hot plume surfaces. The second interval was $100 \mu \mathrm{s}$; it enlarged the surface area of the propagating LIS hot plume. The last interval was $600 \mu \mathrm{s}$; it promoted the interaction between the ignition kernels and the second LIS discharge. The total energy of the laser pulses was fixed at $60 \mathrm{~mJ}$. The characteristics of the hot plume induced by the DPLIS of three pulse intervals were similar to those of the single pulse LIS discharge at a non-reacting air jet condition. Furthermore, the ignition and flame propagation process and induced flow features were also similar 
between the cases of single pulse, $50 \mathrm{~ns}$ dual pulse, and $100 \mu$ s dual pulse although the sizes of ignition kernels were different in each case. Ignition occurred at the mixing laser where the LIS was introduced, and the flame propagated along the mixing layer and the oblique direction from the discharge location. However, the flame propagation in $50 \mathrm{~ns}$ interval case was faster than two other cases. The flame propagation ignited by the LIS discharges was significantly altered when the LIS discharge was induced by the two pulses of $600 \mu$ s interval. The shockwave and hot plume induced by the second laser pulse strongly interacted with the density gradient produced by heat release through the combustion. The rapid lateral propagation of the ignition kernel surfaces occurred, leading to the increased contact surface area between the flame and the mixing layer. As a result, faster flame propagation was achieved. The investigation demonstrated that using DPLIS discharges can be beneficial in igniting and propagating diffusion flames when the pulse intervals of shorter than the electron lifetime scale (under $200 \mathrm{~ns})$ or longer than chemical delay time scale $\left(d t / t_{c d}=1.71\right)$ are selected.

\section{Acknowledgement}

The authors appreciate Dr. Timothy Ombrello at U.S. Air Force Research Laboratory and Dr. Moon Soo Bak at Sungkyunkwan University, South Korea for valuable discussion and comments. Lydia Wermer was supported by U.S. National Science Foundation Graduate Research Fellowship Program (Grant No. DGE-1106756).

\section{References}

[1] T.X. Phuoc, Opt. Commun. 175 (4) (2000) 419-423.

[2] J. Biet, M. Ndem, M. Idir, N. Chaumeix, Combust. Sci. and Technol. 186 (1) (2014) 1-23.

[3] M.S. Bak, M.A. Cappelli, Opt. Express 23 (11) (2015) 419-427.

[4] P.D. Ronney, Opt. Eng. 33 (2) (1994) 510-521.

[5] T.X. Phuoc, Opt. and Laser. Eng. 44 (5) (2006) 351-397. 
[6] T.X. Phuoc, F. P. White, Combust. Flame 119 (3) (1999) 203-216.

[7] J.L. Beduneau, N. Kawahara, T. Nakayama, E. Tomita, Y. Ikeda, Combust. Flame 156 (3) (2009) 642-656.

[8] T.A. Spiglanin, A. Mcilroy, E.W. Fournier, R.B. Cohen, J.A. Syage, Combust. Flame 102 (3) (1995) 310-328.

[9] D. Bradley, C.G.W. Sheppard, I.M. Suardjaja, R. Woolley, Combust. Flame 138 (1) (2004) 55-77.

[10] J.X. Ma, D.R. Alexander, D.E. Poulan, Combust. Flame 112 (4) (1998) 492-506.

[11] T.X. Phuoc, F.P White, Proc. Combust. Inst. 29 (2) (2002) 1621-1628.

[12] C. Dumitrache, A. Yalin, AIAA Paper 2015-2658 (2015).

[13] V.I. Babushok, F.C. DeLucia Jr., J.L. Gottfried, C.A. Munson, A.W. Miziolek, Spectrochim. Acta B 61 (9) (2016) 999-1014.

[14] V. Hohreiter, D. W. Hahn, Spectrochim. Acta B 60 (7) (2005) 968-974.

[15] M.S. Bak, S. Im, M.A. Cappelli, Combust. Flame 161 (7) (2014) 1744-1751.

[16] V. Hohreiter, J.E. Carranza, D.W. Hahn, Spectrochim. ACTA B 59 (3) (2004) 327-333.

[17] M.S. Bak, L. Wermer, S. Im, J. Phys. D Appl. Phys. 48 (48) (2015) 485203.

[18] A.P. Yalin, N. Wilvert, C. Dumitrache, S. Joshi, Phys. Plasmas 21 (10) (2014) 103511. 